\title{
Body Composition and Bone Mineral Density of Collegiate American Football Players
}

\author{
by \\ Hüseyin Hüsrev Turnagöl ${ }^{1}$
}

\begin{abstract}
The aim of this study was to compare whole and segmental body composition and bone mineral density of collegiate American football players by playing positions. Forty collegiate American football players voluntarily participated in this study. Participants were categorized by playing positions into one of five categories i.e., defensive linemen, offensive linemen, defensive secondary players, offensive secondary players and receivers. Whole body composition and bone mineral density were measured by dual x-ray absorptiometry. Offensive and defensive linemen had higher body mass, a body mass index, lean mass and a fat mass index compared to the remaining three positions and a higher lean mass index compared to offensive secondary players and receivers. Offensive linemen had a higher body fat percentage and lower values of upper to lower lean mass than offensive and defensive secondary players and receivers, and higher total mass to the lean mass ratio and fat mass to the lean mass ratio compared to the other players. Offensive linemen had a higher fat mass index and fat mass to the lean mass ratio than defensive linemen. However, in all other measures they were similar. Offensive and defensive secondary players and receivers were similar with respect to the measured variables. Bone mineral density of the players was within the normal range and no difference in lean mass was observed between the legs. In conclusion, findings of this study showed that the total and segmental body composition profile of collegiate American football players reflected the demands of particular playing positions.
\end{abstract}

Key words: Dual $x$-ray absorptiometry, lean mass, fat mass, lean mass index, fat mass index, upper to lower lean mass ratio.

\section{Introduction}

Body composition in athletes is associated with athletic performance (Steweart, 2001) as the proportions of low body fat and high lean body mass provide a good basis for the locomotor activities and specific technical skills required by the sport (Reilly et al., 1996). Thus, the assessment of body composition in athletes by valid and reliable methods is of great importance for planning and evaluation of training programs and nutrient intake over time. American football players have increased body size (Anzell et al., 2011). Indeed, the body mass index (BMI) of football players is often categorized as overweight or obese (Lambert et al., 2012; Steffes et al., 2013). Therefore, body composition of football players influences not only their sport performance, but also their health. In this respect, Steffes et al. (2013) revealed that $6.8 \%$ of college-level football players had the metabolic syndrome, and body fat percent and the BMI were determining factors for metabolic syndrome risk factors. On the other hand, Lambert et al. (2012) showed that categorization of football players' body mass according to the BMI could be misleading due to higher fat free mass of these athletes. Furthermore, recently it has been shown that indicators of fat mass and muscle mass are significant measures of nutritional status and predict health outcomes at all BMI categories (Lang et al., 2015). Thus, the measurement of fat

1 - Division of Nutrition and Metabolism in Exercise, Faculty of Sport Sciences, Hacettepe University, Ankara, Turkey. 
free mass and body fat percent with precise methods is critical. Lean mass is significantly associated with muscle mass, power and speed (Miller et al., 2002). Furthermore, comparison of segmental fat and muscle mass is of great importance in the prevention of sport injuries (Donatelli et al., 2012; Murphy et al., 2013).

Recent methodological advancements in the assessment of body composition enabled researchers to determine fat and lean mass more precisely in athletes. In this regard, the use of dual energy X-ray absorptiometry (DXA) for determining body composition is considered as a gold standard (Bilsborough et al., 2012) as it measures body composition by a 3-compartment model (fat mass, fat free mass and bone mass). Other advantages of DXA are that it is a noninvasive method and enables segmental body composition determination within 5 to 10 minutes by whole body scanning. As a result, there is a growing interest in DXA measurement of athletes' body composition in exercise science. Although, there are numerous studies examining body composition of American football players (Fry and Kraemer, 1991; Gleim 1984; Kraemer et al., 2005), only few of them used DXA (Bosch et al., 2014; Dengel et al., 2014; Lambert et al., 2012; Melvin et al., 2014).

American football is the most popular sport in the USA, thus, there are numerous studies on the physical and physiological characteristics of professional and collegiate football players. These studies have shown that body composition of football players is associated with playing positions (Dengel et al., 2014; Kraemer et al., 2005; Melvin et al., 2014; Pryor et al., 2014) linemen being reported as having the biggest body size. Furthermore, since American football has a long history, studies examining changes in body size and composition of the players over the years are common (Anzell et al., 2013; Dengel et al., 2014; Pryor et al., 2014; Robbins et al., 2013).

In Turkey, American football has a short history of only 24 years. Official leagues of American football in Turkey were established only in 2005. Currently, competitions are held at a professional and collegiate league level. Due to its short history and relatively low interest in this sport in Turkey compared to those of other sport disciplines, particularly in soccer, there is a limited number of studies on the physical and physiological characteristics of American football players. In this regard, none of the studies reporting body composition of American football players in Turkey used DXA. Therefore, there is a need to profile the body composition of American football players with respect to playing positions in Turkey to provide a baseline data for the future.

In addition, having bone mineral density (BMD) within the normal range is of great importance in collision sports, like American football, to prevent or reduce spinal injuries. However, there is no study examining BMD of American football players in Turkey. Therefore, the aim of this study was to compare whole and segmental body composition and BMD of collegiate American football players by playing positions.

\section{Material and Methods}

\section{Participants}

Forty collegiate American football players (age: $21.50 \pm 2.46$ years) voluntarily participated in this study. The subjects were recruited from a college-level team which was the second oldest football team established in Turkey. Mean training experience of the players was $2.51 \pm 1.86$ years and the number of training sessions per week was 3. The subjects were categorized by their playing positions into one of the five categories: defensive linemen ( $D L, n=8)$, offensive linemen $(\mathrm{OL}, \mathrm{n}=7)$, defensive secondary players (DS, $n=9$ ), offensive secondary players (OS, $n=8$ ) and receivers $(\mathrm{R}, \mathrm{n}=8)$. Cornerbacks, line backs and safety positions were grouped into defensive secondary players; quarterbacks and running back players were grouped into offensive secondary players; whereas tight ends and wide receivers were grouped into receivers. Procedures of the study were explained to the participants and their written informed consent was obtained.

\section{Measures}

Anthropometry

Body height was measured by a wall stadiometer (Holtain stadiometer, UK) to the nearest $0.1 \mathrm{~cm}$ and body mass was measured using a scale (Tanita TBF-401A, Germany) to the nearest $0.1 \mathrm{~kg}$. The BMI was calculated as body mass in kilograms divided by the square of body height in meters. 
Hydration Status Assessment.

To monitor hydration status, subjects were asked to collect a midstream sample of urine soon after waking on the morning of the DXA total body scan day. The specific gravity of these urine samples was measured with a manual refractometer (URC-NE; ATAGO Co. Ltd., Tokyo, Japan).

Assessment of Body Composition and Bone Mineral Density

Whole body composition and bone mineral density were measured by a narrow fan bean $\left(4.5^{\circ}\right)$ DXA scanner (Lunar Prodigy Pro; GE Healthcare, Madison, WI, USA) using a total body scan mode. The data was analysed with GE Encore v14.1 software. DXA calibration was completed using phantoms as per manufacturer's standard directions in the morning before the measurements. The scanning mode was automatically selected by the DXA device according to body size. Before each scan, all materials (belts, metal buttons, etc.) that may cause significant attenuation were removed from the DXA measurement room.

All scans and analyses were performed by the same trained and experienced staff. Briefly, after removing the shoes the athlete's body was centered using a centre line on the scanner table as a reference to align the subject. Subjects' hands were turned on sides with thumbs up, palms facing legs and arms were alongside the subject's body so that they were in a midprone position with a standardized gap $(1 \mathrm{~cm})$ between the palms and the trunk. Finally, the head was adjusted $3 \mathrm{~cm}$ below the horizontal line on the table pad and velcro straps were used to secure the subject's knees and feet to prevent movement during the measurement. Participants were urinated and voided their bladder before each scan.

Body composition variables chosen for the present study included four measures of total adiposity, namely the percentage of body fat $(\% \mathrm{BF})$, fat mass (FM), a fat mass index (FMI) and total mass to the lean mass ratio $(\mathrm{BM} / \mathrm{LM})$ and three measures of lean mass, namely, lean mass (LM), a lean mass index (LMI), upper-body lean mass to the lower-body lean mass ratio (U/L ratio). Height-adjusted indexes were calculated as follows: the BMI [body mass $(\mathrm{kg}) /$ height $^{2}\left(\mathrm{~m}^{2}\right)$ ], the $\mathrm{FM}$ index $\left[\mathrm{FMI}=\mathrm{FM}(\mathrm{kg}) /\right.$ height $\left.\left(\mathrm{m}^{2}\right)\right]$, and the
LM index [LMI $=\mathrm{LM}(\mathrm{kg}) /$ height $\left.\left(\mathrm{m}^{2}\right)\right]$. The U/L ratio was calculated as the sum of trunk and arm lean mass divided by leg lean mass. Difference in leg lean mass was calculated as the difference between the left side minus the right side. BMD measures included in this study were whole and spine BMD.

\section{Procedures}

Methods and procedures of the study were approved by the Human Subjects Institutional Review Board at Hacettepe University (No: 16969557-1075). All subjects were tested during the 2013-2014 spring season. The study was carried out at the Laboratory of Nutrition and Metabolism in Exercise at the Faculty of Sport Sciences, Hacettepe University. The subjects were instructed not to engage with heavy exercise and not to take alcohol or caffeine the day before the measurement (Nana et al., 2013). All measurements were performed in the morning after $8-10 \mathrm{~h}$ fasting. Moreover, a euhydration state of athletes was ensured as recommended (Rodriguez-Sanchez and Galloway, 2014).

\section{Statistical Analysis}

Descriptive statistics were calculated as means and standard deviations by playing positions. One way analysis of variance test with the Tukey post hoc test were used to compare means by the position. The level of significance was set as $p<0.05$. Boxplots were used to present median and variation of ten measurements by playing positions (i.e., percent fat, total lean mass, total mass to the lean mass ratio, fat mass to the lean mass ratio, a fat mass index, a lean mass index, difference in lean mass between the legs, upper lean mass to a lower lean mass ratio, whole BMD and spinal BMD).

\section{Results}

Analysis of the data revealed that body mass, height, the BMI and body fat percentages of participants were $88.35 \pm 18.30 \mathrm{~kg}, 179.45 \pm 6.44 \mathrm{~cm}$, $27.42 \pm 4.84 \mathrm{~kg} \cdot \mathrm{m} 2$ and $26.52 \pm 11.38 \%$, respectively. Urine density before DXA measurements was $1019.00 \pm 3.60$ indicating a euhydration state. There was no significant difference with respect to urine density among the playing positions ( $p>0.05$, Table 1) indicating that all subjects underwent DXA measurement at similar hydration levels. Table 1 compares the physical characteristics of 
the subjects by playing positions. There was no significant difference in age, body height and training experience among the groups by their playing positions (Table $1, \mathrm{p}>0.05$ ). Body mass and the BMI of the DL and OL were significantly higher compared with the remaining three positions (Table 1, p $<0.05$ ). According to BMI categories, the DL and OL were classified as moderately obese (BMI, 30-34.9 kg.m2), while other groups were classified as normal (18-24.9 kg.m2).
Table 2 compares the body composition and bone mineral density parameters by playing positions. Figure 1a presents the data for total body fat percent by playing positions. The highest fat percent was determined in the OL (36.17\%) which was significantly higher compared to DS, OS and R positions (Table 2, $\mathrm{p}<0.05$ ). Body fat percent was not different between DL and OL positions $(\mathrm{p}>0.05)$.

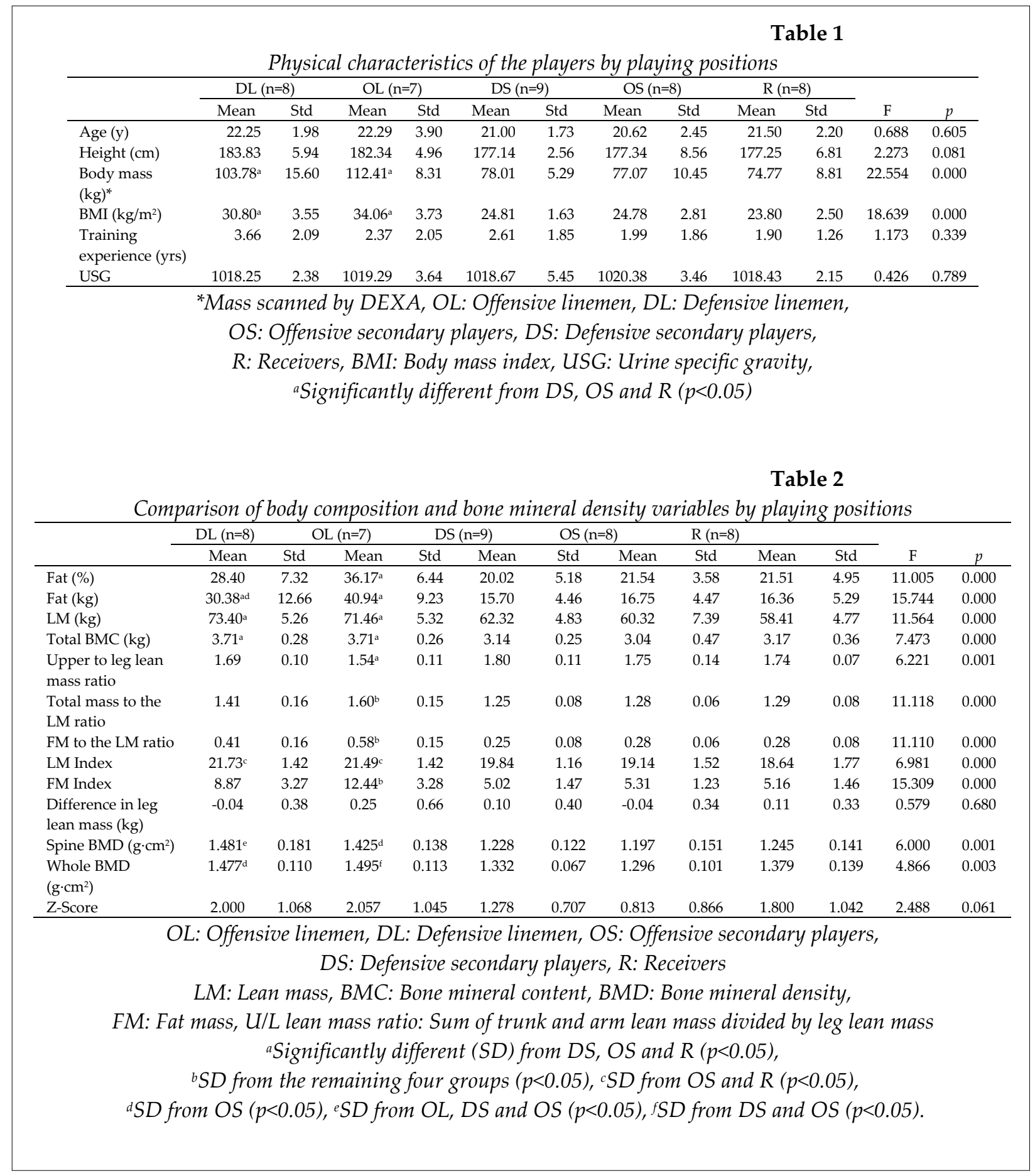



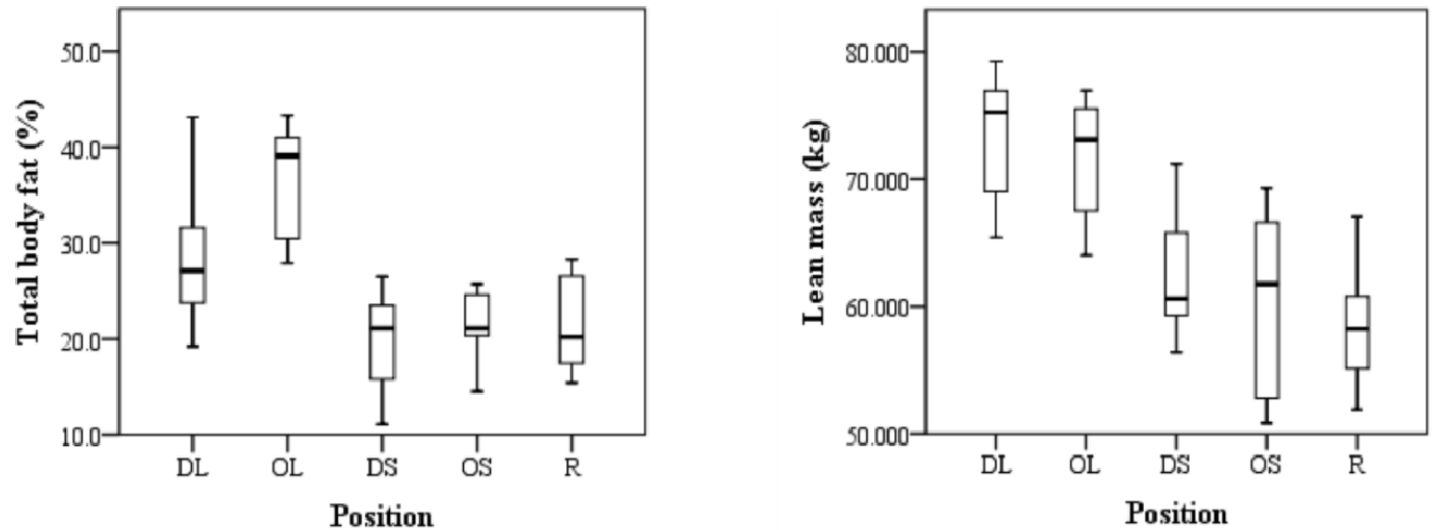

a.

b.
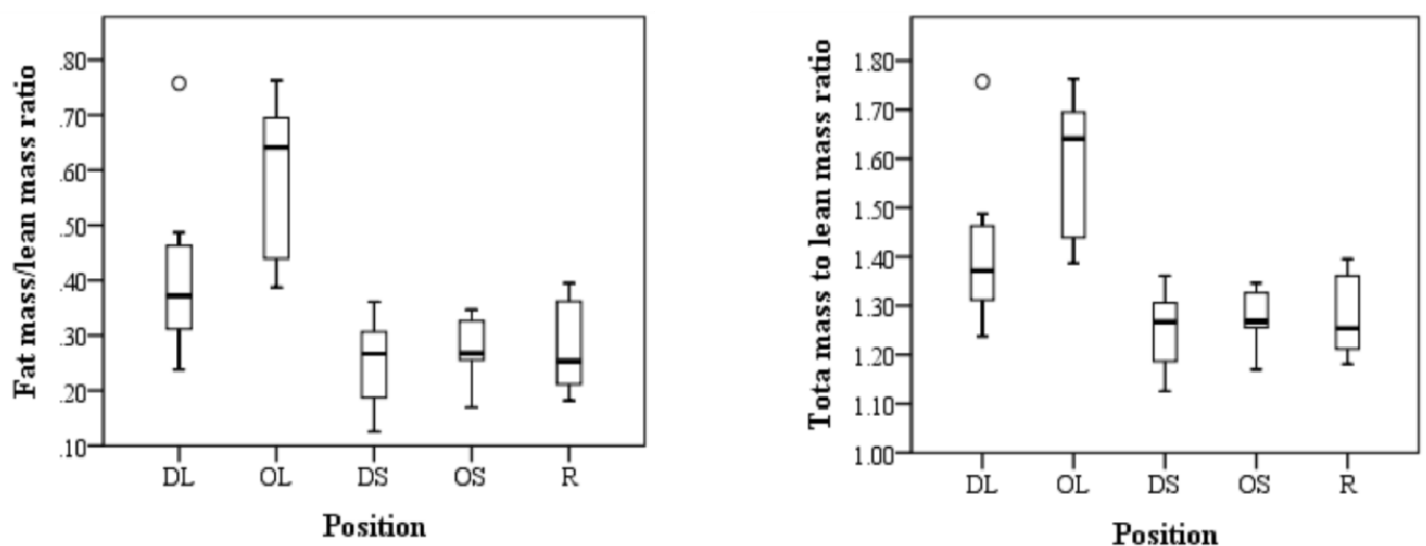

c.

d.
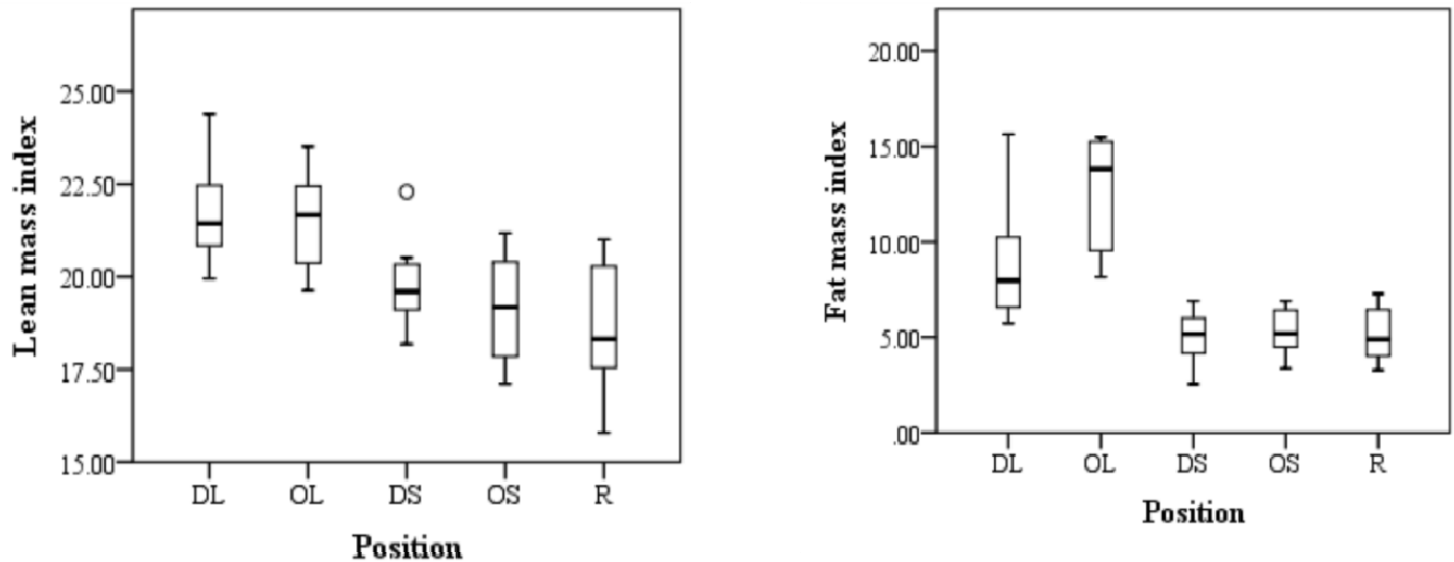

e.

f.

Figure 1a-f.

Boxplots of total body fat, lean mass, fat mass to the lean mass ratio, total mass to the lean mass ratio, lean mass index and fat mass index by playing positions. 


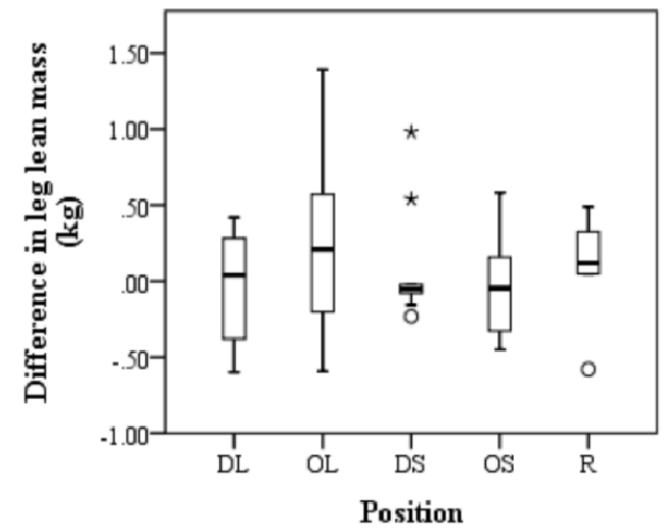

a.

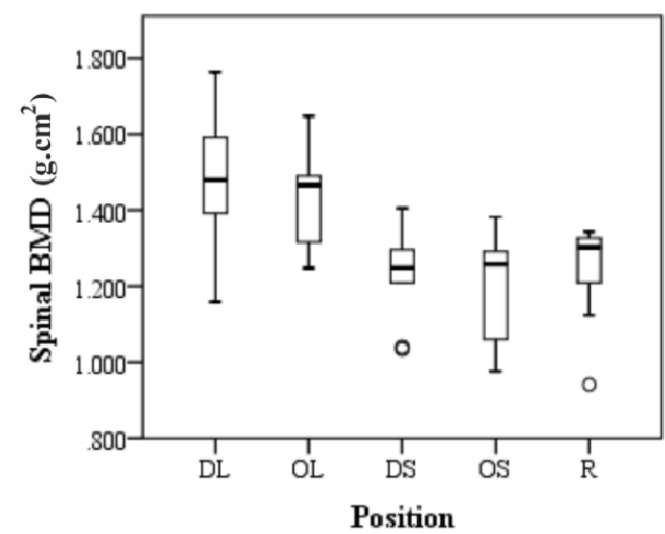

c.

\section{Figure 2a-d.}

Boxplots of difference in right and left leg lean mass, upper to

leg lean mass ratio, spinal bone and whole bone mineral density by playing positions

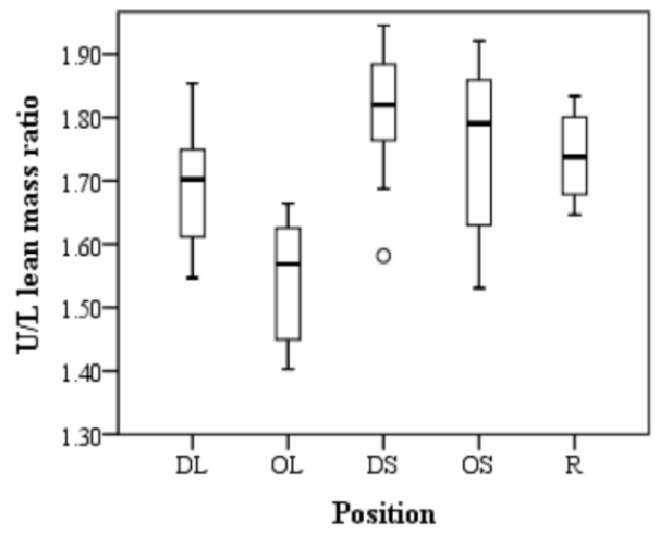

b.

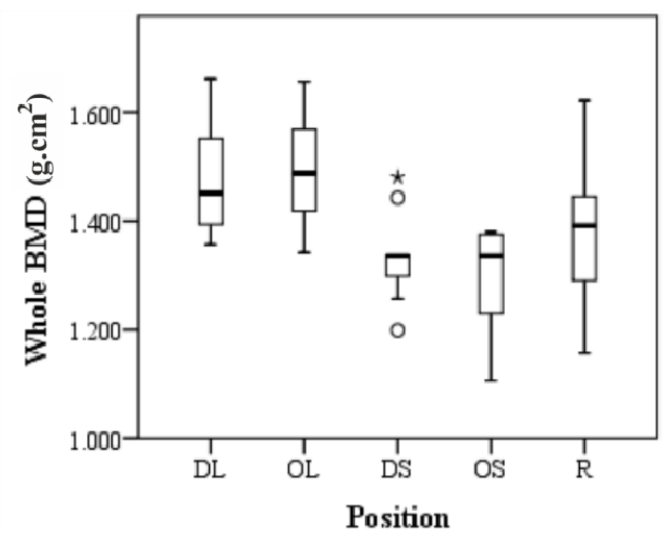

d.

a higher LM index compared to $\mathrm{OS}$ and $\mathrm{R}$ positions $(\mathrm{p}<0.05)$. The OL had a significantly higher fat mass index and fat mass to the LM ratio than the DL. However, in all other measures the DL and OL were similar.

Figures $2 a$ and $2 b$ present the data for the difference in leg lean mass and the U/L lean mass ratio, respectively. There was no significant difference between left and right leg lean mass in any playing position $(\mathrm{p}>0.05)$. DL and OL players had lower values of the U/L lean mass ratio compared to the remaining three positions 
$(\mathrm{p}<0.05)$.

Bone mineral content of the DL and OL were significantly different from DS, $O S$ and $R$ positions $(p<0.05)$. Figures $2 c$ and $2 d$ are the boxplots of the data for spine and whole BMD. Spine BMD of the DL was higher than those of the OL, DS and OS, while the OL had higher spine BMD than the R. The DL and OL had higher whole BMD compared to the other positions, however, only whole BMD in the DL was significantly higher than in the OS, and total BMD of the OL was significantly higher than in DS and OS players $(\mathrm{p}<0.05)$.

\section{Discussion}

To our knowledge, this is the first comprehensive study examining body composition and bone mineral density of American football players in Turkey. There is a growing interest of this sport discipline, yet limited studies are available in the literature reporting body composition characteristics of American football players by using DXA (Bosch et al., 2014; Dengel et al., 2014; Kraemer et al., 2005; Melvin et al., 2014). These studies reported whole and segmental body composition, abdominal and visceral fat accumulation and bone mineral density of football players. As the evaluation of body composition by DXA allows determining regional and whole body fat and lean mass as well as bone mineral density, findings of the present study provide needed baseline data for American football players in Turkey.

Main findings of this study are that OL and DL players were similar with respect to body composition and bone mineral density variables and that players in these two positions were different compared to other positions in most of the examined variables. The OS, DS and R were similar with respect to the measured variables. These findings are in agreement with the literature (Bosch et al., 2014; Dengel et al., 2014; Kraemer et al., 2005; Melvin et al., 2014).

The results of this study showed that players were similar in age, body height and training experience according to the playing position (Table 1, p>0.05). However, DL and OL players had significantly higher body mass and the BMI compared to the remaining three positions (Table $1, \mathrm{p}<0.05$ ). Indeed, DL and OL players were classified as moderately obese (BMI:
30-34.9 kg.m2) according to the BMI categories, while other groups were normal (BMI: 18-24.9 kg.m2). With respect to body fat percentages, although the highest fat percentages were observed in DL (28.40\%) and OL (36.17\%) players, only OL players had a significantly higher body fat percentage compared to DS, OS and R players (Table 2, $\mathrm{p}<0.05$ ). The body fat percentage of DL and OL players was similar ( $p>0.05)$.

These findings are in line with the results of previous studies (Anzell et al., 2013; Dengel et al., 2014; Kraemer et al., 2005; Melvin et al., 2014) in that OL and DL players have a larger body size and a higher body fat percentage compared with other players. Indeed, Dengel et al. (2014) reported that USA national OL and DL players were severely obese (BMI $>35 \mathrm{~kg} \cdot \mathrm{m} 2)$; running backs (RBs), line backers (LBs) and tight ends (TEs) were moderately obese (BMI, 30-34.9 kg.m2) and the remaining positions were normal. Recently, Melvin et al. (2014) reported that the OL and DL had a significantly greater fat percentage, lean mass and fat mass than those of other positions. These findings reflect the demands of the playing positions as the OL and DL players are the anchors and their aim is to keep up their position during the match. Primary duty of the OL and DL players is to block and prevent the other team from scoring, respectively. Similar to the findings of Dengel et al. (2014), DL and OL players in the present study had lower values of upper to leg lean mass (U/L lean mass ratio) compared to the remaining three positions. A lower U/L lean mass ratio increases the stability of the player to maintain his balance and position against the opponent.

Furthermore, DL and OL players had higher LM and the LM index compared to OS and $R$ players $(p<0.05)$. These variables are indicators of the proportions of fat and lean body mass and show that although DL and OL players have a higher body fat percentage and the BMI, the proportion of lean body mass is still high in these players. A higher LM index and LM are associated with large muscle mass and thus, muscle strength.

While, the OL had significantly higher total mass to the LM ratio, the fat mass index and fat mass to the $\mathrm{LM}$ ratio than $\mathrm{DL}$, they were similar in all other measures. These findings are in agreement with the findings of others (Dengel et 
al., 2014; Kraemer et al., 2005; Melvin et al., 2014) representing the demands of the playing position with respect to maintenance of the place.

Body mass, height, and the BMI of OL and DL players in the present study were lower compared to those reported by Dengel et al. (2014). However, the body fat percentage in the present study was higher for all positions except for the OL reported in that study (Dengel et al., 2014). These findings reveal that players of the current study were not well conditioned. This is not surprising as the subjects of the current study were from a collegiate team while Dengel et al. (2014) studied NFL players.

Furthermore, OS, DS and R players were similar with respect to measured variables. These playing positions require more speed, agility and strength to run, catch, and tackle the opponents. This explains why players at these positions had lower total mass to the lean mass ratio and fat mass to the lean mass ratio indicating their high proportion of lean mass. Dengel et al. (2014) reported similar findings for NFL players.

We did not find significant difference between left and right leg lean mass in any position reflecting balanced muscle mass of the players. Unbalance in segmental lean mass may cause skeletal muscle injuries (Donatelli et al., 2012). Similarly, Dengel et al. (2014) did not report segmental lean mass difference in American football players. However, there are outliers in groups, particularly in the OL and DS, what increases the risk of injury for these players.

As known American football is an aggressive physical contact sport involving frequent collisions between players and impact with the ground. Thus, having high bone mineral density is important for the prevention of skeletal injuries, particularly spinal injuries. Spine and total BMD of the players at all playing positions were within the normal range with Z-scores $\geq$ 0.813 (Table 2). With respect to BMC, spine BMD and total BMD, OL and DL players had higher values compared to other playing positions. Though, spine BMD of the DL was significantly higher than this of the OL, DS and OS; moreover, the OL had higher spine BMD than the R (Table 2, $p<0.05)$. The DL and OL had higher total BMD compared to the other positions, however, only total BMD in the DL was significantly higher than in the OS, and total BMD of the OL was significantly higher than in DS and OS players $(\mathrm{p}<0.05)$. It is well known that body mass is positively associated with bone mineral density due to its bone loading effect. Therefore, higher $\mathrm{BMC}$, spine $\mathrm{BMD}$ and total BMD in OL and $\mathrm{DL}$ players compared to the others reflect the bone loading effect of higher body mass. As bone health is significantly affected by genetic and environmental factors, variation in bone health variables among the playing positions was considered as normal.

In conclusion, this is the first study examining collegiate American football players' whole and segmental body composition, and bone mineral density by using DXA with respect to playing positions in Turkey. Significant differences were observed in total and segmental body composition with respect to playing positions which reflect the demands of particular positions. BMD of the players was within the normal range and no difference in lean mass was observed between the legs.

Furthermore, findings of the present study revealed that the body composition profile of collegiate American football players was similar to this in NFL players (Dengel et al., 2014) with OL and DL players having a larger body size compared to other players. This similarity may reflect the intentional drafting and positioning of the players according to their body size (Robbins, 2011) as well as training effects.

This study provides baseline data for body composition and bone mineral density of collegiate American football players in Turkey. However, due to the small number of subjects included in the study, these findings cannot be generalized. Furthermore, again due to the small sample size, we had to combine players together into five groups, what reduces the determination of differences among specific playing positions. Recruitment of more players in future studies may enable researchers to compare players according to their specific playing position and will provide more comprehensive data. Moreover, studies examining relationships between muscle strength, performance indices (speed, power, endurance, agility, etc.) and body composition may improve our understanding of the demands of American football. This would in turn support the selection of players, as well as planning and following the training programs. 


\section{Acknowledgements}

The author thanks to all of the athletes for their participation in the study and to the team captain Alişan Aşar. The author also thanks Süleyman Bulut, Yasemin Güzel and Nilay Kulaksız for their technical support in data collection, and Şükran Nazan Koşar for editing the final version of the manuscript. There was no funding used for this study.

\section{References}

Anzell AR, Potteiger JA, Kraemer WJ, Otieno S. Changes in height, body weight, and body composition in American football players from 1942 to 2011. J Strength Cond Res, 2013; 27: 277-284

Bilsborough JC, Greenway K, Opar D, Livingstone S, Cordy J, Coutts AJ. The accuracy and precision of DXA for assessing body composition in team sport athletes. J Sports Sci, 2014; 32: 1821-8

Bosch TA, Burruss TP, Weir NL, Fielding KA, Engel BE, Weston TD, Dengel DR. Abdominal Body Composition Differences in NFL Football Players. J Strength Cond Res, 2014; 28: 3313-9

Dengel DR, Bosch TA, Burruss TP, Fielding KA, Engel BE, Weir NL, Weston TD. Body composition and bone mineral density of national football league players. J Strength Cond Res, 2014; 28: 1-6

Donatelli R, Dimond D, Holland M. Sport-specific biomechanics of spinal injuries in the athlete (throwing athletes, rotational sports, and contact-collision sports). Clin Sports Med, 2012; 31: $381-96$

Fry AC, Kraemer WJ. Physical performance characteristics of American collegiate football players. J Appl Sport Sci Res, 1991; 5: 126-138

Gleim GW. The profiling of professional football players. Clin Sports Med, 1984; 3: 185-197

Kraemer WJ, Torine JC, Silvestre R, French DN, Ratamess NA, Spiering BA, Hatfield DL, Vingren JL, Volek JS. Body size and composition of National Football League players. J Strength Cond Res, 2005; 19: 485489

Lambert BS, Oliver JM, Katts GR, Green JS, Martin SE, Crouse SF. DEXA or BMI: clinical considerations for evaluating obesity in collegiate division I-A American football athletes. Clin J Sport Med, 2012; 22: 4368

Lang PO, Trivalle C, Vogel T, Proust J, Papazian JP. Markers of metabolic and cardiovascular health in adults: Comparative analysis of DEXA-based body composition components and BMI categories. $J$ Cardiol, 2015; 65: 42-9

Melvin MN, Smith-Ryan AE, Wingfield HL, Ryan ED, Trexler ET, Roelofs EJ. Muscle Characteristics and Body Composition of NCAA Division I Football Players. J Strength Cond Res, 2014; 28: 3320-9

Miller TA, White ED, Kinley K, Congleton JJ, Clark MJ. The effects of training history, player position, and body composition on exercise performance in collegiate football players. J Strength Cond Res, 2002; 16: 44-9

Murphy DF, Connolly DA, Beynnon BD. Risk factors for lower extremity injury: a review of the literature. $\mathrm{Br}$ J Sports Med, 2003; 37: 13-29

Nana A, Slater GJ, Hopkins WG, Burke LM. Effects of exercise sessions on DXA measurements of body composition in active people. Med Sci Sports Exerc, 2013; 45: 178-85

Pryor JL, Huggins RA, Casa DJ, Palmieri GA, Kraemer WJ, Maresh CM. A profile of a National Football League team. J Strength Cond Res, 2014; 28: 7-13

Reilly T, Maughan RJ, Hardy L. Body fat consensus statement of the steering groups of the British Olympic Association. Sports Exerc Injury, 1996; 2: 46-9

Robbins DW. Positional physical characteristics of players drafted into the national football league. J Strength Cond Res, 2011; 25: 2661-2667

Robbins DW, Goodale TL, Kuzmits FE, Adams AJ. Changes in the athletic profile of elite college American 
football players. J Strength Cond Res, 2013; 27: 861-874

Rodriguez-Sanchez N, Galloway SDR. Errors in Dual Energy X-Ray Absorptiometry Estimation of Body Composition Induced by Hypohydration. Int J Sport Nutr Exerc Metab, 2014; 25: 60-8

Steffes GD, Megura AE, Adams J, Claytor RP, Ward RM, Horn TS, Potteiger JA. Prevalence of metabolic syndrome risk factors in high school and NCAA division I football players. J Strength Cond Res, 2013; 27: $1749-57$

Steweart AD. Assessing body composition of athletes. Nutrition, 2001; 17: 694-695

Wilmore JH, Haskell WL. Body composition and endurance capacity of professional football players. J Appl Physiol, 1972; 5: 564-567

\section{Corresponding author:}

\section{Hüseyin Hüsrev TURNAGÖL, PhD}

Division of Nutrition and Metabolism in Exercise, Faculty of Sport Sciences, Hacettepe University, Ankara, Turkey

Phone: +90 5323975259

Fax: +90 3122992167

E-mail address : husrevturnagol@gmail.com; deniz@hacettepe.edu.tr 